\author{
J. Pihlajamäki • M. Kinnunen · E. Ruotsalainen • \\ U. Salmenniemi · I. Vauhkonen • T. Kuulasmaa • \\ S. Kainulainen · M. Laakso
}

\title{
Haplotypes of PPARGC1A are associated with glucose tolerance, body mass index and insulin sensitivity in offspring of patients with type 2 diabetes
}

Received: 8 November 2004 / Accepted: 26 February 2005 / Published online: 24 May 2005

(C) Springer-Verlag 2005

\begin{abstract}
Aims/hypothesis: Decreased expression of the peroxisomal proliferator activated receptor gamma coactivator 1 alpha gene $(P P A R G C 1 A)$ is found in patients with type 2 diabetes, and variants in this gene have been linked with type 2 diabetes. Therefore, we investigated the effects of single nucleotide polymorphisms in PPARGC1A on body composition and glucose tolerance and on insulin sensitivity and secretion. Methods: Non-diabetic offspring ( $n=$ 156, age $34.9 \pm 0.5$ years [mean \pm SEM], BMI $26.2 \pm 0.4 \mathrm{~kg} / \mathrm{m}^{2}$ ) underwent an OGTT and an IVGTT and the hyperinsulinaemic-euglycaemic clamp. The promoter and coding regions of PPARGC1A were sequenced. Results: Two haplotype blocks in PPARGC1A were observed, one in the promoter region $(G-1774 A, A-1679 G, T-1422 C, A-1278 G$, $C-543 A$ ) and one in the coding region and $3^{\prime}$ regions (Thr394Thr, Asp475Asp, Gly482Ser, Thr528Thr, Thr612 Met, $\mathrm{G}+2381 \mathrm{~A})$. The coding region haplotype carrying the rare allele in codons 482 and 528 was associated with elevated glucose levels in an OGTT ( $p=0.024$, adjusted for age, sex and BMI) and a haplotype carrying the rare alleles in codons 394 and 475 was associated with low BMI $(p=0.033)$, high rates of whole-body glucose uptake $(p=0.045)$ and low glucose levels in the OGTT $(p=0.037)$. Conclusions/interpretation: We conclude that PPARGC1A is likely to contribute to the risk of diabetes in offspring of patients with type 2 diabetes.
\end{abstract}

J. Pihlajamäki and M. Kinnunen contributed equally to this study

J. Pihlajamäki $(\bowtie) \cdot$ M. Kinnunen $\cdot$ E. Ruotsalainen

U. Salmenniemi - I. Vauhkonen · T. Kuulasmaa · M. Laakso

Department of Medicine,

University of Kuopio,

P.O. Box 1627, 70210 Kuopio, Finland

e-mail: pihlajam@uku.fi

Tel.: +358-17-173311

Fax: $+358-17-162445$

S. Kainulainen

Department of Radiology,

University of Kuopio,

Kuopio, Finland
Keywords Haplotypes · Insulin resistance $\cdot$ Insulin secretion $\cdot$ PGC- $1 \alpha \cdot$ PPARGC1A . Type 2 diabetes . Visceral obesity

Abbreviations QTDT: a computer program - SNP: single nucleotide polymorphism - WBGU: whole-body glucose uptake

\section{Introduction}

Type 2 diabetes is caused by a combination of environmental factors and inherited defects in insulin action and secretion. A transcriptional coactivator of the nuclear receptor peroxisome proliferator activated receptor gamma $(\mathrm{PGC}-1 \alpha)$ and the gene encoding it (PPARGC1A) play a role in adaptive thermogenesis and insulin sensitivity [1-3]. Down-regulation of PPARGC1A expression has been observed in patients with type 2 diabetes and their firstdegree relatives, which has been suggested to be linked to the risk of type 2 diabetes due to impaired fat oxidation $[4,5]$. Polymorphisms in codons 475 and 482 of PPARGC1A have been linked with type 2 diabetes [2], insulin resistance [3] and the rates of lipid oxidation [6].

In this study we screened the promoter region and the coding region of PPARGC1A in 156 middle-aged non-diabetic offspring of patients with type 2 diabetes to identify single nucleotide polymorphisms (SNPs). Additionally, we investigated the effect of these SNPs and haplotypes on BMI, glucose tolerance, insulin sensitivity and insulin secretion measured by the hyperinsulinaemic-euglycaemic clamp and the IVGTT.

\section{Subjects and methods}

Subjects All subjects participating in the study were of Finnish ancestry. The study population and protocol have been previously described in detail [7]. On day 1 all subjects underwent an OGTT to evaluate their glucose tolerance according to the World Health Organization 1998 
criteria. All subjects had normal liver, kidney and thyroid function, no history of excessive alcohol intake and no severe chronic diseases. Informed consent was obtained from all subjects after the purpose and potential risks of the study were explained to them. The protocol was approved by the Ethics Committee of the University of Kuopio and was in accordance with the Helsinki Declaration.

Metabolic studies On day 2, metabolic studies were performed after an overnight fast. First, an IVGTT was performed to determine the first-phase insulin secretion capacity. Samples for the measurement of blood glucose and plasma insulin were drawn at $-5,0,2,4,6,8,10,20$, $30,40,50$ and $60 \mathrm{~min}$. Immediately after the IVGTT, insulin sensitivity was evaluated by the hyperinsulinaemiceuglycaemic clamp technique (insulin infusion of 240 pmol $\cdot \mathrm{kg}^{-1} \cdot \mathrm{min}^{-1}$ ). The mean rates of glucose infusion during the second hour of the clamp were used to calculate the rates of insulin-stimulated whole-body glucose uptake (WBGU).

Body composition and abdominal fat distribution Body composition was determined by bioelectrical impedance (RJL Systems, Detroit, MI, USA) and abdominal fat distribution by computed tomography (Siemens Volume Zoom, Germany) at the level of the fourth lumbar vertebra.

Genotyping Templates for sequencing $2.5 \mathrm{~kb}$ of the promoter region and variants in the coding region were amplified by PCR. Primers were planned according to the available sequences (Genbank accession no. NC_000004 for the chromosome 4 and for PPARGC1A mRNA NM 013261) and are available from the authors (J. P.). Sequencing was performed with BigDye Terminator v1.1 Cycle Sequencing Kit using the ABI-PRISM 3100 Genetic Analyser (Applied Biosystems, Foster City, CA, USA). The 3' region was investigated with an additional SNP (rs3774923, $A+2381 G$ ) using the TaqMan Allelic Discrimination Assays (Applied Biosystems).

Statistical analyses Basic analysis of the data was performed with the SPSS/Win programs (version 10.0; SPSS, Chicago, IL, USA). Haplotype frequencies were estimated and likely haplotypes were reconstructed for each individual using the MERLIN program (http://www.sph.umich. edu/csg/abecasis/Merlin). The effect of each haplotype on the quantitative parameters was analysed with the familybased test of linkage disequilibrium using the QTDT program with age, sex and BMI as covariates, when appropriate [8]. A value of $p<0.05$ was considered to be statistically significant. All data are presented as means \pm SEM.

\section{Results}

The 156 study subjects (70 men and 86 women) were middle-aged (age $34.9 \pm 0.5$ years), their BMI was slightly above the normal range $\left(26.2 \pm 0.4 \mathrm{~kg} / \mathrm{m}^{2}\right)$ and they were non-diabetic on the basis of the OGTT (133 were normoglycaemic and 23 had IGT). Linkage disequilibrium was observed between the variants in the promoter region and between the variants in the coding region and the $3^{\prime}$ region ( $\mathrm{D}^{\prime}>90 \%$ in both regions, Table 1 ). Three most common haplotypes explained more than $80 \%$ of the variation in the $5^{\prime}$ region (haplotype 1a: 11211 [31\%]; haplotype 1b: 12111 [28\%]; haplotype 1c: 21111 [24\%]), and coding and $3^{\prime}$ regions (haplotype $1 \mathrm{~b}: 111111$ [44\%]; haplotype 2b: 112211 [29\%]; haplotype 3b: 221111 [7\%]) of PPARGC1A. Genotype frequencies did not differ significantly from the Hardy-Weinberg equilibrium.

The Asp475Asp variant was associated with BMI (26.5 $0.5 \mathrm{~kg} / \mathrm{m}^{2}$ in subjects homozygous for the wild-type vs $24.2 \pm 0.4 \mathrm{~kg} / \mathrm{m}^{2}$ in subjects who were heterozygous, $p=$ $0.031), 2$-h glucose in an OGTT $(6.42 \pm 0.12$ vs $5.68 \pm 0.25$

Table 1 The degree of linkage disequilibrium ( $R^{2}$ value) between the variants in the promoter, the coding region and the $3^{\prime}$ region of PPARGC1A

\begin{tabular}{|c|c|c|c|c|c|c|c|c|}
\hline & SNP code & Frequency & & & & & & \\
\hline Promoter & & & G-1774A & A-1679G & $\mathrm{T}-1422 \mathrm{C}$ & A-1278G & C-543A & - \\
\hline$G-1774 A$ & rs2970869 & 0.28 & - & - & - & - & - & - \\
\hline$A-1679 G$ & - & 0.33 & $0.193 * * *$ & - & - & - & - & - \\
\hline$T-1422 C$ & rs2970870 & 0.37 & $0.225 * * *$ & $0.294 * * *$ & - & - & - & - \\
\hline$A-1278 G$ & rs7695542 & 0.02 & 0.008 & 0.000 & 0.011 & - & - & - \\
\hline$C-543 A$ & - & 0.02 & 0.006 & $0.032 * *$ & 0.008 & 0.000 & - & - \\
\hline Coding and $3^{\prime}$ regions & & & Thr394Thr & Asp475Asp & Gly482Ser & Thr528Thr & Thr612Met & $\mathrm{A}+2381 \mathrm{G}$ \\
\hline Thr394Thr & rs2970847 & 0.13 & - & - & - & - & - & - \\
\hline Asp475Asp & - & 0.08 & $0.503 * * *$ & - & - & - & - & - \\
\hline Gly482Ser & rs8192678 & 0.38 & $0.085 * * *$ & $0.045 * *$ & - & - & - & - \\
\hline Thr528Thr & rs 3755863 & 0.38 & $0.101 * * *$ & $0.053 * *$ & $0.819 * * *$ & - & - & - \\
\hline Thr612Met & rs 3736265 & 0.10 & $0.021^{*}$ & 0.005 & $0.058 * *$ & $0.069 * * *$ & - & - \\
\hline $\mathrm{A}+2381 \mathrm{G}$ & rs3774923 & 0.07 & 0.001 & 0.009 & $0.025 * *$ & $0.029 * *$ & $0.427 * * *$ & - \\
\hline
\end{tabular}

No significant linkage disequilibrium was observed between variants in different blocks (promoter vs coding region blocks) SNP Single nucleotide polymorphism $* p \leq 0.05, * * p \leq 0.01, * * * p<0.001$ 
Table 2 The effect of the most common haplotypes of PPARGC1A on BMI, abdominal fat distribution by computed tomography (CT), fasting plasma (fP) glucose and glucose AUC during the OGTT, insulin levels during the first $10 \mathrm{~min}$ of the IVGTT, and on the rates of whole-body glucose uptake (WBGU) during the hyperinsulinaemic clamp in 156 offspring of patients with type 2 diabetes (QTDT program analysis adjusted for age, sex and BMI)

\begin{tabular}{|c|c|c|c|c|c|c|c|}
\hline & \multirow[t]{2}{*}{$\mathrm{BMI}\left(\mathrm{kg} / \mathrm{m}^{2}\right)$} & \multicolumn{2}{|c|}{ Abdominal fat CT } & \multicolumn{2}{|l|}{ OGTT } & \multirow{2}{*}{$\begin{array}{l}\text { IVGTT } \\
\text { (insulin AUC } \\
{\left[\mathrm{pmol} \cdot 1^{-1} \cdot \min ^{-1}\right] \text { ) }}\end{array}$} & \multirow{2}{*}{$\begin{array}{l}\text { Hyperinsulinaemic } \\
\text { clamp }\left(\mathrm{WBGU}\left[\mu \mathrm{mol} \cdot \mathrm{kg}^{-1}\right.\right. \\
\left.\left.\text { lean body mass } \cdot \mathrm{min}^{-1}\right]\right)\end{array}$} \\
\hline & & $\begin{array}{l}\text { S.c. } \\
\left(\mathrm{cm}^{2}\right)\end{array}$ & $\begin{array}{l}\text { Visceral } \\
\left(\mathrm{cm}^{2}\right)\end{array}$ & $\begin{array}{l}\text { fP glucose } \\
(\mathrm{mmol} / \mathrm{l})\end{array}$ & $\begin{array}{l}\text { Glucose AUC } \\
\left(\mathrm{mmol} \cdot 1^{-1} \cdot \min ^{-1}\right)\end{array}$ & & \\
\hline \multicolumn{8}{|l|}{ Promoter } \\
\hline Haplotype 1a & $27.4 \pm 0.3$ & $253 \pm 7$ & $104 \pm 6$ & $5.1 \pm 0.1$ & $847 \pm 18$ & $1,944 \pm 182$ & $55.5 \pm 17.0$ \\
\hline Haplotype $2 \mathrm{a}$ & $26.7 \pm 0.4$ & $250 \pm 8$ & $109 \pm 6$ & $5.2 \pm 0.1$ & $859 \pm 19$ & $1,979 \pm 190$ & $54.6 \pm 17.3$ \\
\hline Haplotype $3 \mathrm{a}$ & $26.1 \pm 0.5$ & $256 \pm 8$ & $102 \pm 7$ & $5.1 \pm 0.1$ & $855 \pm 20$ & $2,003 \pm 208$ & $58.3 \pm 16.3$ \\
\hline \multicolumn{8}{|c|}{ Coding and $3^{\prime}$ regions } \\
\hline Haplotype $1 b$ & $26.2 \pm 0.5$ & $268 \pm 9$ & $102 \pm 8$ & $5.1 \pm 0.1$ & $843 \pm 16$ & $2,012 \pm 214$ & $57.7 \pm 17.8$ \\
\hline Haplotype $2 b$ & $26.1 \pm 0.3$ & $259 \pm 9$ & $106 \pm 8$ & $5.3 \pm 0.1$ & $868 \pm 21^{*}$ & $1,739 \pm 222$ & $56.4 \pm 18.4$ \\
\hline Haplotype $3 b$ & $24.3 \pm 0.4^{*}$ & $261 \pm 13$ & $108 \pm 12$ & $5.2 \pm 0.1$ & $779 \pm 32 *$ & $2,059 \pm 336$ & $60.9 \pm 15.6^{*}$ \\
\hline
\end{tabular}

Values are means \pm SEM

$* p<0.05$ in QTDT analysis against subjects without the studied haplotype. For haplotype definition, see text

$\mathrm{mmol} / \mathrm{l}, p=0.007)$ and the rates of WBGU $(55.3 \pm 1.5 \mathrm{vs}$ $61.1 \pm 2.9 \mu \mathrm{mol} \cdot \mathrm{kg}^{-1}$ lean body mass $\cdot \mathrm{min}^{-1}, p=0.042$ ) in QTDT analysis. The association with 2-h glucose remained statistically significant after the adjustment for age, sex and BMI $(p=0.025)$. None of the SNPs was associated with fasting glucose or blood pressure. With the exception of the Asp475Asp, none of the other SNPs were associated significantly with BMI, glucose tolerance or insulin sensitivity.

Next, we analysed the effect of the haplotypes on BMI, glucose tolerance, insulin sensitivity and secretion. Table 2 shows that the third haplotype in the coding region (haplotype $3 \mathrm{~b}$ ), carrying the codon 475 variant, was associated with low BMI ( $p=0.033$ adjusted for age and sex). The same haplotype was also associated with low 2-h glucose $(p=0.005)$ and glucose AUC $(p=0.019)$ in the OGTT and high rates of WBGU ( $p=0.045$, Table 2$)$. The difference in glucose AUC remained statistically significant after the adjustment for BMI $(p=0.037)$ in the entire study population and in the normoglycaemic subjects $(p=0.021)$. The haplotype $2 \mathrm{~b}$ in the coding region carrying the rare alleles in codons 482 and 528 was associated with high glucose AUC in the OGTT ( $p=0.024$, adjusted for age, sex and BMI). This haplotype tended to be associated with IGT (14 subjects with the haplotype had IGT vs nine subjects with NGT, $18 \%$ vs $10 \%$, respectively, $p=0.065$ ). No effect of the haplotypes was found on insulin AUC in the OGTT, abdominal fat distribution and insulin secretion (measured as insulin AUC during the first $10 \mathrm{~min}$ of the IVGTT or as an insulinogenic index in the OGTT) with or without adjustment for insulin action.

\section{Discussion}

Low expression of PPARGC1A has been associated with IGT and type 2 diabetes [4, 5], and variants in PPARGC1A have been linked with type 2 diabetes $[2,3,6]$. In this study we showed that haplotypes in the coding region of
PPARGC1A were associated with glucose tolerance, BMI and insulin sensitivity in offspring of type 2 diabetic patients, suggesting that PPARGC1A may contribute to the risk of type 2 diabetes.

In previous studies the Gly482Ser polymorphism and the silent polymorphisms Thr394Thr and Asp475Asp of PPARGC1A have been associated with type 2 diabetes [2, $3]$. We observed one haplotype block in the $5^{\prime}$ region and another in the coding region and the $3^{\prime}$ region of the gene, as previously observed in an Austrian study [9]. In our study, the haplotype carrying the rare alleles in codons 482 and 518 (haplotype 2b) was associated with high glucose AUC in the OGTT. Similarly, a haplotype carrying the rare allele in codon 482, along with the common allele in codon 394, was associated with type 2 diabetes in Japanese subjects [3]. Furthermore, our results from the STOP-NIDDM trial indicate that the rare 482Ser allele increases the risk of type 2 diabetes in subjects with IGT [10]. In the Austrian study, a protective haplotype carrying the common alleles of variants in codons 394, 482 and 528 of PPARGC1A was related to lower risk of type 2 diabetes and highest insulin secretory response in the OGTT. Although we could not replicate this finding in our study, we did show that haplotype $3 \mathrm{~b}$, which carries the rare allele in codons 394 and 475 and the common allele in codon 482, was associated with low BMI and glucose AUC in the OGTT and better insulin sensitivity. Thus, some of the PPARG $C 1 A$ haplotypes may be protective. An explanation for discrepant results could be that variants more distal in these haplotypes confer the risk. In addition, the effect on type 2 diabetes risk may be relatively small, and therefore could not be observed in all studies. Finally, multiple testing could be a potential problem and some of observed associations may be false positives.

In conclusion, we observed that haplotypes in the coding region of PPARGC1A are associated with glucose tolerance, BMI and insulin sensitivity in offspring of patients with type 2 diabetes. These haplotypes could either in- 
crease the risk or be protective against diabetes. The results strengthen the evidence that low PPARGC1A expression in patients with type 2 diabetes and their relatives could be explained by an inherited variation in the PPARGC1A locus.

Acknowledgements This study was financially supported by grants from the Academy of Finland, the Finnish Diabetes Research Foundation, the EVO-fund of the Kuopio University Hospital and the European Union (to M. L., QLG1-CT-1999-00674). We thank laboratory technicians Leena Uschanoff, Kaija Eirola and Anna-Lisa Gidlund for genotyping.

\section{References}

1. Puigserver P, Wu Z, Park CW, Graves R, Wright M, Spiegelman BM (1998) A cold-inducible coactivator of nuclear receptors linked to adaptive thermogenesis. Cell 92:829-839

2. Ek J, Andersen G, Urhammer SA et al (2001) Mutation analysis of peroxisome proliferator-activated receptor-gamma coactivator-1 (PGC-1) and relationships of identified amino acid polymorphisms to type II diabetes mellitus. Diabetologia 44: 2220-2226

3. Hara K, Tobe K, Okada T et al (2002) A genetic variation in the PGC-1 gene could confer insulin resistance and susceptibility to type II diabetes. Diabetologia 45:740-743
4. Patti ME, Butte AJ, Crunkhorn S et al (2003) Coordinated reduction of genes of oxidative metabolism in humans with insulin resistance and diabetes: potential role of PGC1 and NRF1. Proc Natl Acad Sci USA 100:8466-8471

5. Mootha VK, Lindgren CM, Eriksson KF et al (2003) PGC1alpha-responsive genes involved in oxidative phosphorylation are coordinately downregulated in human diabetes. Nat Genet 34:267-273

6. Muller YL, Bogardus C, Pedersen O, Baier L (2003) A Gly482Ser missense mutation in the peroxisome proliferator-activated receptor gamma coactivator-1 is associated with altered lipid oxidation and early insulin secretion in Pima Indians. Diabetes 52:895-898

7. Salmenniemi U, Ruotsalainen E, Pihlajamäki J et al (2004) Multiple abnormalities in glucose and energy metabolism and coordinated changes in levels of adiponectin, cytokines, and adhesion molecules in subjects with metabolic syndrome. Circulation 110:3842-3848

8. Abecasis GR, Cardon LR, Cookson WO (2000) A general test of association for quantitative traits in nuclear families. Am J Hum Genet 66:279-292

9. Oberkofler H, Linnemayr V, Weitgasser R et al (2004) Complex haplotypes of the PGC-1alpha gene are associated with carbohydrate metabolism and type 2 diabetes. Diabetes 53:1385-1393

10. Andrulionyte L, Zacharova J, Chiasson JL, Laakso M (2004) Common polymorphisms of the PPAR-gamma2 (Pro12Ala) and PGC-1alpha (Gly482Ser) genes are associated with the conversion from impaired glucose tolerance to type 2 diabetes in the STOP-NIDDM trial. Diabetologia 47:2176-2184 